\title{
Virtual-bound, filamentary and layered states in a box-shaped quantum dot of square potential form the exact numerical solution of the effective mass Schrödinger equation
}

\author{
A. Luque , A. Mellor, I. Tobías, E. Antolín , P.G. Linares, I. Ramiro, A. Martí
}

\begin{abstract}
A B S T R A C T
The effective mass Schrödinger equation of a QD of parallelepipedic shape with a square potential well is solved by diagonalizing the exact Hamiltonian matrix developed in a basis of separation-of-variables wavefunctions. The expected below bandgap bound states are found not to differ very much from the former approximate calculations. In addition, the presence of bound states within the conduction band is confirmed. Furthermore, filamentary states bounded in two dimensions and extended in one dimension and layered states with only one dimension bounded, all within the conduction band-which are similar to those originated in quantum wires and quantum wells - coexist with the ordinary continuum spectrum of plane waves. All these subtleties are absent in spherically shaped quantum dots, often used for modeling.
\end{abstract}

\section{Introduction}

Quantum dots (QDs) are mesoscopic structures for which ab initio modeling, often used for bulk crystals, is difficult to apply [1] because they require extensive utilization of computational resources. The $\mathrm{k} \cdot \mathrm{p}$ method may [2-4] provide a procedure for dealing approximately with these problems. However, this method can also be very costly in the use of computational resources [5] when considering mesoscopic structures. Although the cooperation of specialists in quantum calculations and device technology is very desirable, and is sometimes achieved [6,7], the development of simple models, easier to use by experimentalists assists technological progress effectively.

In this regard, the use of the effective mass approximation (actually a 1-band variant of the $\mathrm{k} \cdot \mathrm{p}$ method) may be interesting for devices where the optical interaction between the $Q D$ bound states and the conduction band (CB) is essential. This is, among others, the case for intermediate band solar cells (IBSC) [8-11] and QD infrared photodetectors [12,13].

The simplicity of the model is still increased, and widely used, if square well potentials are considered. These potentials are usually the band offset (corrected by strain) between the different semiconductors forming the nanostructure, which for this purpose are considered of sharp edges. In this paper we want to show how despite this simplicity the solutions can present high complexity whose full discussion is facilitated by the simplicity of the basic model. In particular we want to show the appearance in QDs of virtual bound states (confined states within the $C B$ ) already discussed by several authors [5,14-16], filamentary and layered states.

In this context, QDs have often been modeled as spherical potential wells [17,18], in particular when the actual QDs have this shape. Spherical symmetry has also been used to determine the optimal size of the QDs in IBSC [19] in which the electron confinement was produced by the band offset. Since the spherical geometry permits analytical solutions which are textbook exercises in quantum mechanics [20], it has also been used even if the symmetry was known not to be spherical [21,22]. However, in many other cases, and in particular when the QDs are grown by molecular beam epitaxy (MBE) in the Stranski-Krastanov mode, e.g., in the commonly used InAs QDs grown in GaAs, the QDs take the shape of short quadrangular truncated pyramids that may be approximated as a parallelepipedic box [23-27] (of dimensions $2 a \times 2 a \times 2 c$ in this paper).

The use of the box shape in the QD allows approximate solution of the time independent Schrödinger equation (TISE) by separation-ofvariables: the eigenfunctions $\Phi(x, y, z)=\xi(x) \psi(y) \zeta(z)$ are the product of three one-dimensional functions, each one being eigenfunctions of a one-dimensional Hamiltonian, in which the $\mathrm{QD}$ is characterized by a square potential well.

This model is useful not only to describe the $\mathrm{CB}$ electrons but also to determine, by application of the appropriate effective masses and band offsets, the energies of VB electrons in all the 
three bands (heavy holes, light holes and split-off band) [27] that characterize these electrons. For the $\mathrm{CB}$, the method provides a reasonable approximation of the eigenfunctions and thus it can be directly applied (much more easily than in spherical symmetry) to the determination of the intraband photon absorption matrix elements $[25,26,28]$. In the case of the VB, a four-band $\mathrm{k} \cdot \mathrm{p}$ solution, still based in the box shaped symmetry, is necessary $[27,29]$ for determination of the eigenfunctions and the interband absorption coefficient.

However, the separation-of-variables solutions are only an approximation [25]. In this paper we shall use these solutions as a basis for the development of the described Hamiltonian so obtaining the exact eigenvalues and eigenfunctions; exact, of course, in the limits of our geometrical description of the QD, its square potential and in the frame of the one-electron treatment and the effective mass approximation.

Section 2 presents the exact Hamiltonian we want to solve and describes how it is modified to accept separation-of-variables solutions. A perturbation Hamiltonian is then defined that transforms the latter into the former. Section 3 analyzes the nature of the eigenvectors and eigenvalues, describing the modalities of the discrete spectrum and the different continua spectra found and finally calculates the eigenfunctions and eigenvectors for each modality. Section 4 studies the density of states associated to each discrete or continuum spectrum modality. Section 5 compares the spherical symmetry solutions to the box shaped symmetry used in this paper. Finally Section 6 draws some conclusions.

\section{The exact and separation-of-variables potentials}

\subsection{The Schrödinger equation}

The time-independent Schrödinger equation (TISE) is

$$
\frac{\hbar^{2}}{2 m^{*}} \frac{\nabla^{2} \Psi}{\Psi}+E=V(\boldsymbol{r})
$$

where the right side term is the band edge position (with changed sign if it refers to holes). The asterisk indicates that an effective mass is used. The energy origin is arbitrary and for the calculations the zero is set at the bottom of the potential well inside the $\mathrm{QD}$ (which is at the dot material conduction band edge) and $U$ outside it. In symbolic language ( $\forall=$ for all, $\wedge=$ and, $\vee=$ or),

$V(\boldsymbol{r})=\left\{\begin{array}{l}0 \forall|x|<a \wedge|y|<a \wedge|z|<c \\ U \forall|x| \geq a \vee|y| \geq a \vee|z| \geq c\end{array}\right.$

However, for presentation purposes, the zero shall be set at the barrier material conduction band edge, which means that $U$ must be subtracted from all the energy results. The reason for taking the origin of potential at the QD CB bottom is explained in the two following sections.

\subsection{One dimensional solutions}

The functions $\xi(x), \psi(y), \zeta(z)$ are defined by

$$
\frac{\hbar^{2}}{2 m^{*}} \frac{d^{2} \xi / d x^{2}}{\xi}+E_{x}= \begin{cases}0 & \forall|x|<a \\ U & \forall|x| \geq a\end{cases}
$$

and similarly for $\psi(y), \zeta(z)$ (in the latter case using $c$ instead of $a$ as the well boundary).

The position of the origin of potential at the $\mathrm{QD} \mathrm{CB}$ bottom is common in textbooks for one dimensional square potential wells. It also fits with our choice in the last section.

Finding the solutions for $\xi(x)$ (or for $\psi(y), \zeta(z)$ ) constitutes a simple exercise of differential equations. In this context, a discussion is provided e.g. in [25]. For $E<U$ (the subindex $x$ is dropped in this subsection) bounded solutions, different from the trivial $\xi(x)=0$, are even $(\cos (k x))$ or odd $(\sin (k x))$ harmonic functions inside the well flanked by fading exponential functions outside it $(\exp (-\kappa x) \forall x \geq a)$. Solutions may only exist for certain values of the wavenumber $k_{n}$ for which the non-fading exponential solution is canceled. The energy $E_{n}$, fading coefficient $\kappa_{n}$ and $k$-values are related by

$E_{n}=\hbar^{2} k_{n}^{2} / 2 m^{*}=U-\hbar^{2} \kappa_{n}^{2} / 2 m^{*}$

The index $n$ denotes the different permitted energies in increasing order. It is a quantum number (QN). Odd QNs correspond to even functions and vice versa.

Table 1 presents the values of $k$ for the different QNs and the one-dimensional energy. The $\mathrm{CB}$ offset and $\mathrm{QD}$ dimensions are those in [28] and are derived from the data of a prototype IBSC in [30] (sample SB).

Although the effective mass is different for the dot (InAs) and barrier (GaAs) material, we use the dot material value across this paper. A straightforward modification of Eq. (1) where $m^{*}$ is position-variable would lead to a non-hermitical Hamiltonian [31]. There are several possible modifications but their discussion are beyond the scope of this paper. This effective mass choice is accurate for low QNs and less so for the extended states to be studied below, but still very meaningful qualitatively.

For $E \geq U$, the solution is harmonic with wavenumber $k$ inside the potential well and also harmonic, even or odd, outside it but with a different value of the wavenumber $k_{e}$ and a phase term. That is, they are of the form $\cos \left(k_{e} x-\theta\right)$ or $\sin \left(k_{e} x-\theta\right)$. Details can be found, e.g. in [28]. In this case,

$E_{n}=U+\hbar^{2} k_{e}^{2} / 2 m^{*}=\hbar^{2} k^{2} / 2 m^{*}$

and

$\left(k_{e} / k\right) \cot (k a)=\cot \left(k_{e} a-\theta\right)$
$\left(k_{e} / k\right) \tan (k a)=\tan \left(k_{e} a-\theta\right)$

respectively for the even and odd functions.

For $E \geq U, k_{e}$ can take any value and therefore it leads to a continuum spectrum of energies. Since the mathematics of continuum spectra is rather complicated, it is common to assume that the wavefunctions are restricted to a large but finite region (a segment of length $2 L$, with large $L$, for one-dimensional cases, or a big parallelepiped for three-dimensional ones) and assume periodic conditions there. This leads [28] to

$k_{e} L-\theta=\tilde{n} \pi / 2$

where $\tilde{n}$ is an integer, odd for the even solutions and even for the odd solutions.

Neglecting the variation of $\theta$ the permitted values of $k_{e x}$ are separated $\Delta k_{e} \cong \pi / 2 L$ that is small as long as $L$ is big (with respect to $a$ or $c$ ). However, only a numerable set of $k_{e}$-values are now permitted and the new QN, $\tilde{n}$, has now appeared.

Table 1

Values of $k$ (multiplied by the potential well half-width) and one-dimensional energy for the $\mathrm{CB}$ offset and QD dimensions in [28]. Energies are with respect to

\begin{tabular}{|c|c|c|c|c|c|c|}
\hline \multirow[t]{2}{*}{$n$} & \multicolumn{4}{|c|}{$x-y$-eigenfunctions } & \multicolumn{2}{|c|}{$z$-eigenfunctions } \\
\hline & 1 & 2 & 3 & 4 & 1 & 2 \\
\hline$k_{x} a, k_{z} c$ & 1.299 & 2.579 & 3.806 & 4.810 & 0.968 & 1.695 \\
\hline$E_{0}(\mathrm{eV})$ & -0.439 & -0.338 & -0.180 & -0.0045 & -0.338 & -0.059 \\
\hline
\end{tabular}
the barrier material $\mathrm{CB}$ bottom.

$U=0.473 \mathrm{eV} ; m^{*}=0.0294 m_{e} ; a=8 \mathrm{~nm} ; c=3 \mathrm{~nm}$. 
Due to the hermitical property of the Hamiltonian, any two eigenfunctions, bound or extended, are strictly orthogonal. For easier handling, the eigenfunctions must be normalized. This is easy for bound functions (it may be calculated analytically). Strictly speaking, extended states cannot be normalized (at least in the ordinary sense) but this difficulty is circumvented by integrating the square of the eigenfunction's absolute value in the interval $(-L,+L)$. If outside the QD the harmonic function has amplitude one, this integration is approximately $L$ (and the wavefunction norm is $L^{1 / 2}$ ) if $L$ is much larger than the QD dimensions. $L$ it is arbitrarily chosen but any measurable magnitude has, under this model, an expression containing $L$ that cancels out the $L$ dependence.

\subsection{The perturbation potential}

By adding the one-dimensional Eq. (3) corresponding to the three coordinates we obtain

$\frac{\hbar^{2}}{2 m^{*}} \frac{\nabla \Phi}{\Phi}+E_{n_{x}, n_{y}, n_{z}}=V_{0}(\boldsymbol{r})$

where

$\Phi(x, y, z)=\xi(x) \psi(y) \zeta(z)$

$E_{n_{x}, n_{y}, n_{z}}=E_{x, n_{x}}+E_{y, n_{y}}+E_{z, n_{z}}$

The choice of the origin of potentials at the QD CB bottom, both in the one three-dimensional TISE Eq. (1) and the one dimensional ones Eq. (3) allows for the simple expression of the energies in Eq. (9), without caring for readjusting potential references.

However, $V_{0}(\boldsymbol{r})$ is different from $V(\boldsymbol{r})$. As explained in [25], it is the same inside the QD and outside it in front of the faces, but it takes the value $2 U$ in front of the edges and of $3 U$ in front of the corners. In symbolic language,

$V_{0}(\boldsymbol{r})=\left\{\begin{array}{l}0 \forall|x|<a \wedge|y|<a \wedge|z|<c \\ U \forall|x| \geq a \wedge|y|<a \wedge|z|<c \\ U \forall|x|<a \wedge|y| \geq a \wedge|z|<c \\ U \forall|x|<a \wedge|y|<a \wedge|z| \geq c \\ 2 U \forall|x| \geq a \wedge|y| \geq a \wedge|z|<c \\ 2 U \forall|x| \geq a \wedge|y|<a \wedge|z| \geq c \\ 2 U \forall|x|<a \wedge|y| \geq a \wedge|z| \geq c \\ 3 U \forall|x| \geq a \wedge|y| \geq a \wedge|z| \geq c\end{array}\right.$

Thus, the exact Hamiltonian can be written as

$H=-\frac{\hbar^{2}}{2 m^{*}} \nabla^{2}+V_{0}(\boldsymbol{r})+V^{\prime}(\boldsymbol{r})=H_{0}+V^{\prime}(\boldsymbol{r})$

where the (improperly called) perturbation potential is

$$
V^{\prime}(\boldsymbol{r})=V(\boldsymbol{r})-V_{0}(\boldsymbol{r})=\left\{\begin{array}{l}
O \forall|x|<a \wedge|y|<a \wedge|z|<c \\
O \forall|x| \geq a \wedge|y|<a \wedge|z|<c \\
0 \forall|x|<a \wedge|y| \geq a \wedge|z|<c \\
0 \forall|x|<a \wedge|y|<a \wedge|z| \geq c \\
-U \forall|x| \geq a \wedge|y| \geq a \wedge|z|<c \\
-U \forall|x| \geq a \wedge|y|<a \wedge|z| \geq c \\
-U \forall|x|<a \wedge|y| \geq a \wedge|z| \geq c \\
-2 U \forall|x| \geq a \wedge|y| \geq a \wedge|z| \geq c
\end{array}\right.
$$

\section{Matrix representation of the exact Hamiltonian}

In this section the exact Hamiltonian is going to be represented as a matrix in basis of separation-of-variables wavefunctions and then diagonalized.

\subsection{Three dimensional basis states}

The separation-of-variables wavefunctions $\left|n_{x}, n_{y}, n_{z}\right\rangle=\xi_{n_{x}} \psi_{n_{y}}$ $\zeta_{n_{z}}=\Phi_{n_{x}, n_{y}, n_{z}}$ are the eigenfunctions of the Hamiltonian $H_{0}$. Each one-dimensional eigenfunction may be bound or extended. To distinguish this fact we can write $\left|\hat{n}_{x}, \tilde{n}_{y}, \hat{n}_{z}\right\rangle$ where $\hat{n}_{x}$ indicates that the $x$-eigenfunction is bound and $\tilde{n}_{y}$ denotes that the $y$-eigenfunction is extended. The $H_{0}$ eigenvalues are in Eq. (9) whose components may be found in Eqs. (4) or (5). As said before, we usually subtract $U$ from the values obtained to refer the energies to the barrier material $\mathrm{CB}$ bottom.

The set $\left|n_{x}, n_{y}, n_{z}\right\rangle$ forms an orthonormal basis that nevertheless is somewhat involved. It is formed of purely bound states $\left|\hat{n}_{x}, \hat{n}_{y}, \hat{n}_{z}\right\rangle$ (OE states), of purely extended states $\left|\tilde{n}_{x}, \tilde{n}_{y}, \tilde{n}_{z}\right\rangle$ (3E states) and of states that are mixed, with a single extended state, of the type $\left|\hat{n}_{x}, \hat{n}_{y}, \tilde{n}_{z}\right\rangle$ (and all the circular permutations: $1 \mathrm{Ez}, 1 \mathrm{Ex}$, 1 Ey states) and with a two extended states $\left|\tilde{n}_{x}, \tilde{n}_{y}, \hat{n}_{z}\right\rangle$ (and all the circular permutations: 2Exy, 2Eyz, 2Ezx states). The QN corresponding to the extended states is linked, as indicated in Eq. (7) to a certain value of $L$ and it may be physically more meaningful to use $k_{e}$ and the parity as wavefunction definer although for calculations we shall use the corresponding QN (the closest one of the same parity, once $L$ is given).

Examples of $\mathrm{HO}$ eigenfunctions of the $\mathrm{OE}, 1 \mathrm{E}$ and $2 \mathrm{E}$ sets are given in Fig. 1.

Note that for extended states the electron tends to be outside the QD.

If $H_{0}$ is developed in this basis, it forms a diagonal matrix, although the ordering of the diagonal elements in this matrix is complicated. For instance we can start by ordering the finite number of states of the bound $\mathrm{OE}$ states and then put the $3 \mathrm{E}$ states, but they are infinite, and we still have to situate the $2 \mathrm{E}$ and 1 E states.

\subsection{Integration in regions}

As derived from the perturbation potential in Eq. (12), the matrix elements of the perturbation Hamiltonian $\left\langle n_{x, 1}, n_{y, 1}, n_{z, 1}\right|$ $V^{\prime}(\boldsymbol{r})\left|n_{x, 2}, n_{y, 2}, n_{z, 2}\right\rangle$ require the integrations of wavefunctions restricted to a certain region of the space and multiplied by a multiple of $U$. This section is devoted to the calculation of these integrations.

The complex conjugation necessary in quantum mechanics for the internal products and matrix elements is not used here because all the functions are real.

In Table 2 and 3 the values of the integration inside and outside the QD are presented for bound one-dimensional eigenfunctions in the $\mathrm{x}$ and $\mathrm{z}$ coordinates respectively. The column to the left represents the internal product; for different QNs it must be zero. The very small values observed in some cases are to be considered the noise background of our calculations. For small QNs, the probability of finding the electron outside the QD (look at the cases with equal QNs) is very small, so justifying the choice of the QD effective mass. For large QNs it may be more spread. When the parity of the QNs is different, the product of wavefunctions is odd and its integral is zero inside the QD. Outside it, it takes different signs for positive and negative abscissas. When the QNs are different, but of the same parity, the integrals inside and outside the QD balance out. 
a

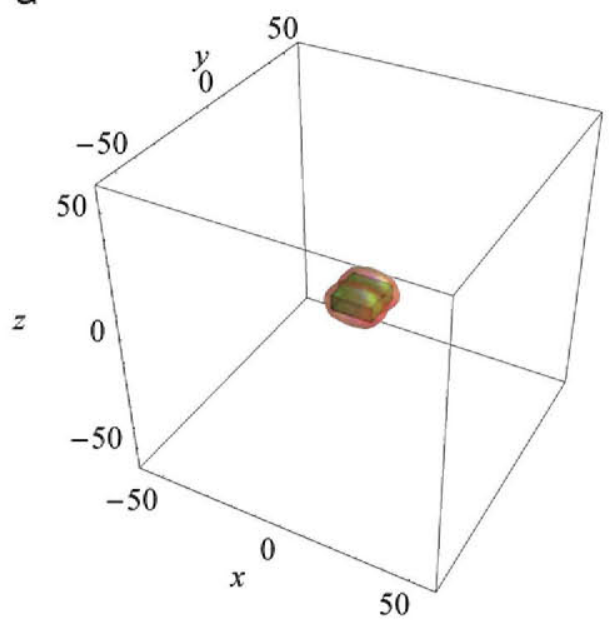

b

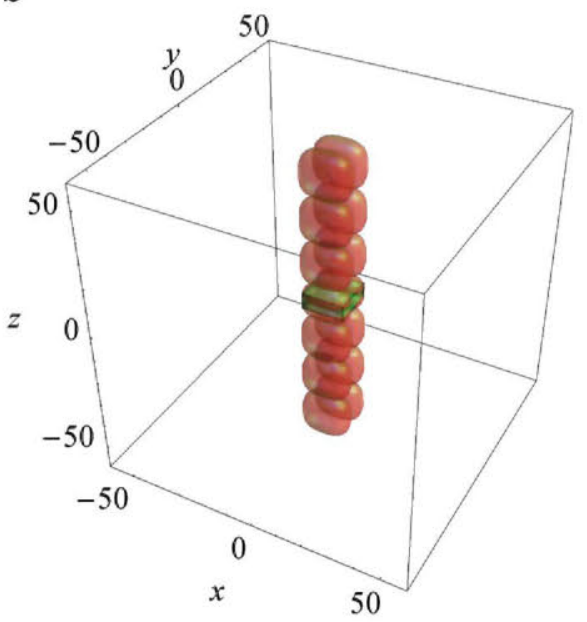

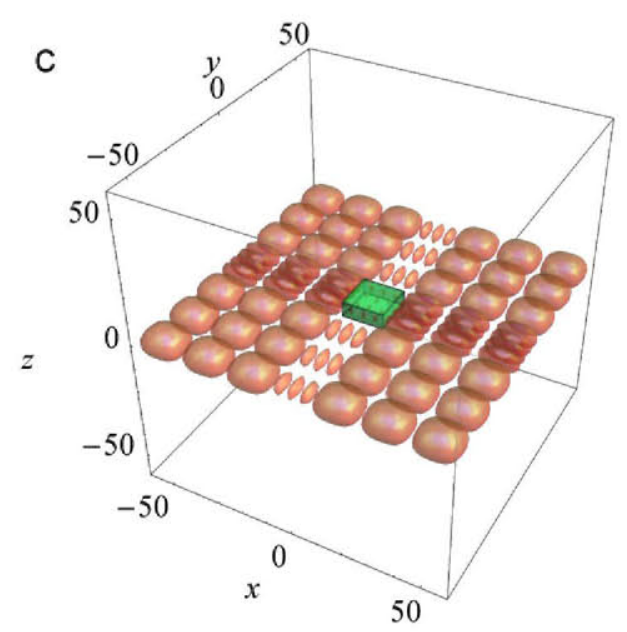

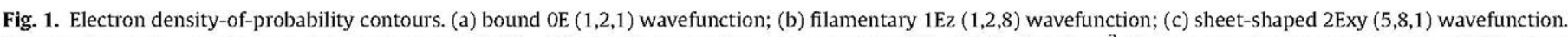

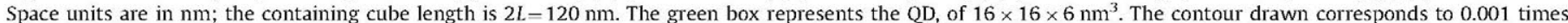

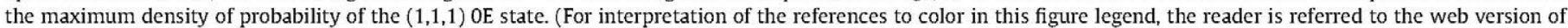
this article.)

Table 2

Integration in regions of bound one-dimensional eigenfunctions corresponding to the coordinates $x$ or $y$.

\begin{tabular}{llclc}
\hline QNs & $\int_{-\infty}^{\infty} \xi_{\hat{n}_{x}} \xi_{\hat{n}_{x}^{\prime}} d x$ & $\int_{-\infty}^{a} \xi_{n_{x}} \xi_{\hat{n}_{x}^{\prime}} d x$ & $\int_{-a}^{a} \xi_{\hat{n}_{x}} \xi_{\hat{n}_{x}^{\prime}} d x$ & $\int_{a}^{\infty} \xi_{\hat{n}_{x}} \xi_{\hat{n}_{x}^{\prime}} d x$ \\
\hline 1,1 & 1 & 0.00638387 & 0.987232 & 0.00638387 \\
1,2 & 0 & 0.0133362 & 0 & -0.0133362 \\
1,3 & $1.606 \times 10^{-16}$ & -0.0217575 & 0.0435151 & -0.0217575 \\
1,4 & 0 & -0.0267906 & 0 & 0.0267906 \\
2,2 & 1 & 0.0279778 & 0.944044 & 0.0279778 \\
2,3 & 0 & -0.0461097 & 0 & 0.0461097 \\
2,4 & $-3.47531 \times 10^{-17}$ & -0.059098 & 0.118196 & -0.059098 \\
3,3 & 1 & 0.0779097 & 0.844181 & 0.0779097 \\
3,4 & 0 & 0.111237 & 0 & -0.111237 \\
4,4 & 1 & 0.336536 & 0.326929 & 0.336536 \\
\hline
\end{tabular}

Table 3

Integration in regions of bound one-dimensional eigenfunctions corresponding to the coordinate $z$.

\begin{tabular}{clllc}
\hline QNs & $\int_{-\infty}^{\infty} \zeta_{\hat{n}_{z}} \zeta_{\hat{n}_{z}^{\prime}} d z$ & $\int_{-\infty}^{c} \zeta_{\hat{n}_{z}} \zeta_{\hat{n}_{z}^{\prime}} d z$ & $\int_{-c}^{c} \zeta_{\hat{n}_{z}} \zeta_{\hat{n}_{z}^{\prime}} d z$ & $\int_{c}^{\infty} \zeta_{\hat{n}_{z}} \zeta_{\hat{n}_{z}^{\prime}} d z$ \\
\hline 1,1 & 1 & 0.000375021 & 0.99925 & 0.000375021 \\
1,2 & 0 & 0.00328518 & 0 & -0.00328518 \\
2,2 & 1 & 0.0345654 & 0.930869 & 0.0345654 \\
\hline
\end{tabular}

For extended one-dimensional eigenfunctions of different QNs the orthogonality is theoretically required and well verified in our calculations as well. Thus, for $L \rightarrow \infty$ (the amplitude of $\xi_{\tilde{n}_{x}}$ is rendered zero by the normalization),

$\int_{-a}^{a} \xi_{\tilde{n}_{x}} \xi_{\tilde{n}_{x}^{\prime}} d x=0$
$\int_{-\infty}^{-a} \xi_{\tilde{n}_{x}} \xi_{\tilde{n}_{x}^{\prime}} d x+\int_{a}^{\infty} \xi_{\tilde{n}_{x}} \xi_{\tilde{n}_{x}^{\prime}} d x=\delta_{\tilde{n}_{x}, \tilde{n}_{x}^{\prime}}$

and for bound/extended eigenfunctions

$\int_{-a}^{a} \xi_{\tilde{n}_{x}} \xi_{\tilde{n}_{x}} d x=0$

$\int_{-\infty}^{-a} \xi_{\hat{n}_{x}} \xi_{\tilde{n}_{x}} d x+\int_{a}^{\infty} \xi_{\hat{n}_{x}} \xi_{\tilde{n}_{x}} d x=0$

The $y$ - and $z$-eigenfunctions behave similarly.

3.3. The exact Hamiltonian matrix elements, eigenvalues and eigenvectors

The exact Hamiltonian matrix when developed in eigenfunctions of the Hamiltonian $H_{0}$ of Eq. (11) is the sum of the matrix 
representation of $H_{0}$, which is a diagonal matrix, and the matrix representation of $V$, described in Eq. (12).

The matrix elements of the latter are

$$
\begin{aligned}
& \left\langle n_{x}, n_{y}, n_{z}\left|V^{\prime}\right| n_{x}^{\prime}, n_{y}^{\prime}, n_{z}^{\prime}\right\rangle= \\
& -U\left[\begin{array}{l}
\int_{-a}^{a} \xi_{n_{x}} \xi_{n_{x}^{\prime}} d x\left(\int_{-\infty}^{-a} \psi_{n_{y}} \psi_{n_{y}^{\prime}} d y+\int_{a}^{\infty} \psi_{n_{y}} \psi_{n_{y}} d y\right)\left(\int_{-\infty}^{-c} \zeta_{n_{z}} \zeta_{n_{z}^{\prime}} d z+\int_{c}^{\infty} \zeta_{n_{z}} \xi_{n_{x}^{\prime}} d x+\int_{a}^{\infty} \xi_{n_{x}^{\prime}} d z\right)+ \\
\left(\int_{-\infty}^{-a} \xi_{n_{x}} \xi_{n_{x}} d x\right) \int_{-a}^{a} \psi_{n_{y}} \psi_{n_{y}^{\prime}} d y\left(\int_{-\infty}^{-c} \zeta_{n_{z}} \zeta_{n_{z}} d z+\int_{c}^{\infty} \zeta_{n_{x}} \xi_{n_{x}} \zeta_{n_{z}^{\prime}} d x\right)\left(\int_{-\infty}^{-a} \psi_{n_{y}} \psi_{n_{y}^{\prime}} d y+\int_{a}^{\infty} \psi_{n_{y}} \psi_{n_{y}^{\prime}} d y\right) \int_{-c}^{c} \zeta_{n_{z}} \psi_{n_{z}^{\prime}} d z
\end{array}\right] \\
& -2 U\left[\left(\int_{-\infty}^{-a} \xi_{n_{x}} \xi_{n_{x}} d x+\int_{a}^{\infty} \xi_{n_{x}} \xi_{n_{x}^{\prime}} d x\right)\left(\int_{-\infty}^{-a} \psi_{n_{y}} \psi_{n_{y}^{\prime}} d y+\int_{a}^{\infty} \psi_{n_{y}} \psi_{n_{y}^{\prime}} d y\right)\right. \\
& \left.\times\left(\int_{-\infty}^{-c} \zeta_{n_{z}} \zeta_{n_{z}} d z+\int_{c}^{\infty} \zeta_{n_{z}} \zeta_{n_{z}} d z\right)\right]
\end{aligned}
$$

where no distinction has been made between bound and extended states.

According to Eq. (14), the matrix elements linking a bound and an extended wavefunction in a given coordinate are zero. Therefore, the only non-zero matrix elements are those belonging to the same set (OE, 3E etc.) of those enumerated in Section 3.1. This is the same as saying that the Hamiltonian matrix is the direct sum of the Hamiltonian matrices in these sets. In other words, it is a diagonal of blocs, although many of these blocs are of infinite dimension.

\subsubsection{Purely bound OE states}

For the dimensions and potential of our prototype cell there are $4 \mathrm{QNs}$ for the $x$ and $y$ one-dimensional eigenfunctions and 2 for the $z$ one-dimensional eigenfunction. Thus, there are $32 \mathrm{H}_{0}$ eigenfunctions for bound states. The eigenvalues are calculated with Eq. (9) using Table 1. They are represented in Fig. 2(a). The energy first-order approximation

$E_{1}=E_{0}+\left\langle n_{x}, n_{y}, n_{z}\left|V^{\prime}\right| n_{x}, n_{y}, n_{z}\right\rangle$

is also presented. In the preceding equation $E_{0}$ is used as a short notation for what in Eq. (8) is denoted as $E_{n_{x}, n_{y}, n_{z}}$.

States with $n_{x} \neq n_{y}$ are double degenerated. Notice that the first order approximation moves all the states downward. This shift is very little for the low energies but substantial for the high energies.

An exact calculation of the perturbed Hamiltonian, that is, the exact one within the model limitations, can be obtained by

a

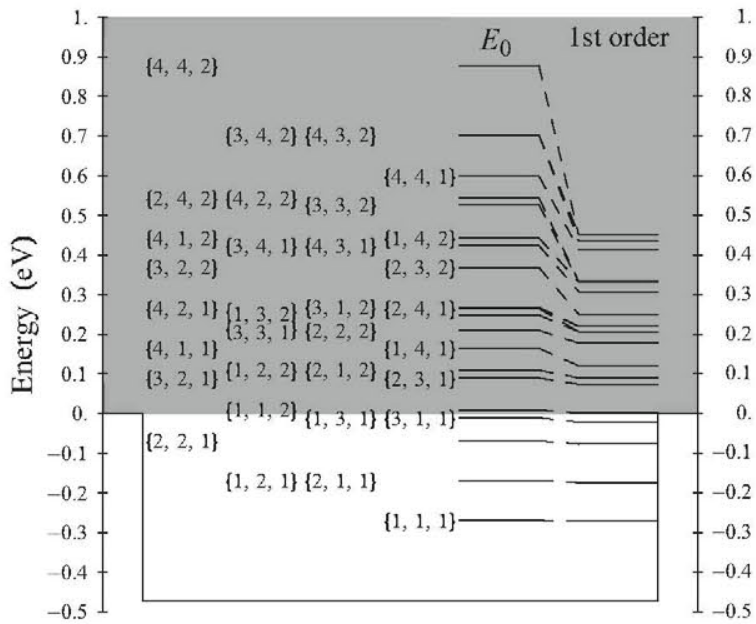

calculating the matrix

$\left\langle n_{x}, n_{y}, n_{z}|H| n_{x}^{\prime}, n_{y}^{\prime}, n_{z}^{\prime}\right\rangle=\left\langle n_{x}, n_{y}, n_{z}\left|H_{0}\right| n_{x}^{\prime}, n_{y}^{\prime}, n_{z}^{\prime}\right\rangle+\left\langle n_{x}, n_{y}, n_{z}\left|V^{\prime}\right| n_{x}^{\prime}, n_{y}^{\prime}, n_{z}^{\prime}\right\rangle$

and obtaining (with Mathematica) the eigenvalues and eigenvectors. Of course, $\left\langle n_{x}, n_{y}, n_{z}\left|H_{0}\right| n_{x}^{\prime}, n_{y}^{\prime}, n_{z}^{\prime}\right\rangle$ is the diagonal matrix of elements equal to $E_{0}$.The eigenvalues are represented in Fig. 2(b) side by side with the 1st order approximations (which are also in part (a) of the figure) for easy comparison. Changes are small but visible for the high energies. It must be stressed that, for the exact calculations, the energy levels cannot be related to any set of QNs. In fact, the eigenstates are linear combinations of the unperturbed Hamiltonian eigenstates that are the product of onedimensional wavefunctions, each with one QN. This relationship is represented in Fig. 3. The absolute value of the coefficient of

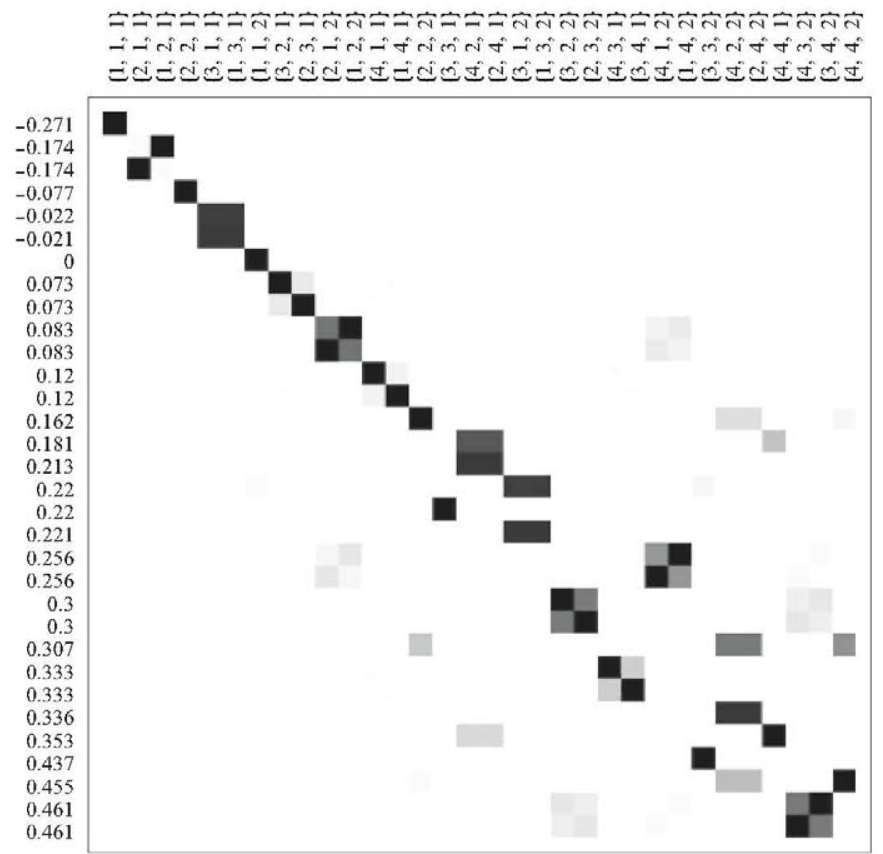

Fig. 3. Strength of the projection of the eigenstates corresponding to the exact Hamiltonian eigenenergy, to the left, on the $H_{0}$ Hamiltonian eigenstates (above). Black, full projection; white, zero projection. Eigenstates are characterized by their energy (left, in eV).

b

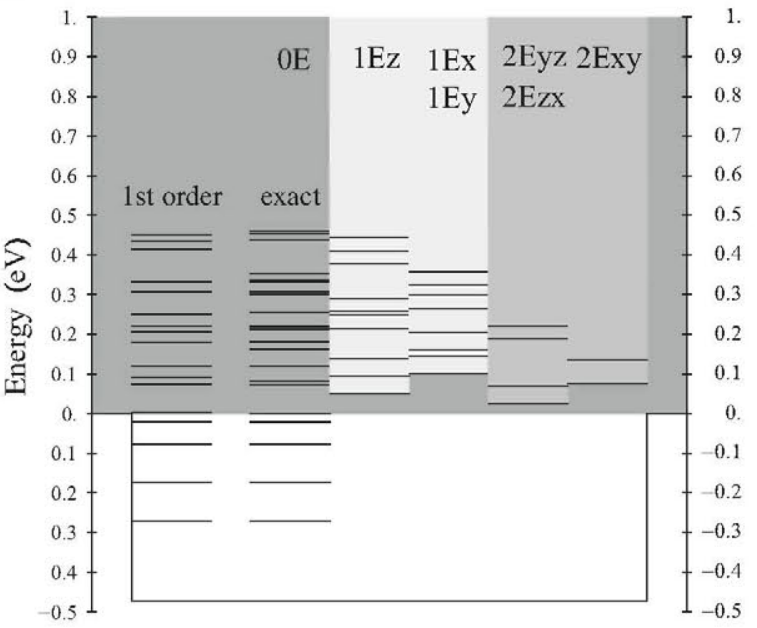

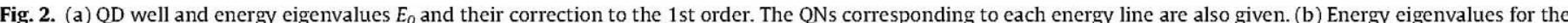

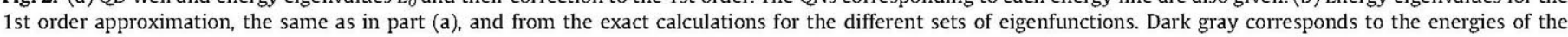

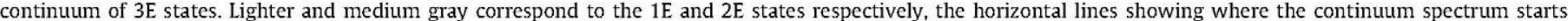
above the energy of the bound part of the wavefunctions. One continum of energies starts above each horizontal line in these two groups of sets. 
$\left|n_{x}, n_{y}, n_{z}\right\rangle$ is represented in a gray scale (1 black; 0 , white). The numeric values are given in the Supplementary materials.

It can be seen that, in general, there are a small number of dominant unperturbed states corresponding to each exact state. Diagonal blocks of four boxes are often found sometimes corresponding to double degenerate states. The order of the unperturbed eigenstates on the top follows the order of the increasing $E_{0}$ energies.

\subsubsection{One dimensional extended $1 E$ states}

This set is actually separated into three sets, $1 \mathrm{Ez}, 1 \mathrm{Ex}$ and $1 \mathrm{Ey}$, corresponding respectively to the states extended in $z, x$ and $y$ and bound in the remaining variables. Let us examine first the $1 \mathrm{Ez}$ states. The eigenvalues of $H_{0}$ are, according to Eqs. (11) and (5),

$E_{0}=E_{x}+E_{y}+\left(U+\hbar^{2} k_{e z}^{2} / 2 m^{*}\right)-U=E_{00}+\hbar^{2} k_{e z}^{2} / 2 m^{*}$

Note the energy in this equation is with respect to barrier material $\mathrm{CB}$ bottom; this results from subtracting $U$ from the energies with respect to the $\mathrm{QD} C \mathrm{CB}$ bottom (the two Us cancel). The second zero subindex in $E_{00}$ indicates that $k_{c z}$ is zero.

The matrix elements have now two bound and one extended function; that is, using Eq. (13) they are

$\left\langle\hat{n}_{x}, \hat{n}_{y}, \tilde{n}_{z}\left|V^{\prime}\right| \hat{n}_{x}^{\prime}, \hat{n}_{y}^{\prime}, \tilde{n}_{z}^{\prime}\right\rangle=\left\langle\hat{n}_{x}, \hat{n}_{y}\left|V^{\prime}\right| \hat{n}_{x}^{\prime}, \hat{n}_{y}^{\prime}\right\rangle \delta_{\tilde{n}_{z}, \tilde{n}_{z}^{\prime}}$ where

$$
\begin{aligned}
& \left\langle\hat{n}_{x}, \hat{n}_{y}\left|V^{\prime}\right| \hat{n}_{x}^{\prime}, \hat{n}_{y}^{\prime}\right\rangle= \\
& -U \times\left[\begin{array}{c}
\left.\int_{-a}^{a} \xi_{n_{x}} \xi_{n_{x}^{\prime}} d x\left(\int_{-\infty}^{-a} \psi_{n_{y}} \psi_{n_{y}^{\prime}} d y+\int_{a}^{\infty} \psi_{n_{y}} \psi_{n_{y}^{\prime}} d y\right)+\right] \\
\left(\int_{-\infty}^{-a} \xi_{n_{x}} \xi_{n_{x}^{\prime}} d x+\int_{a}^{\infty} \xi_{n_{x}} \xi_{n_{x}^{\prime}} d x\right) \int_{-a}^{a} \psi_{n_{y}} \psi_{n_{y}^{\prime}} d y
\end{array}\right] \\
& -2 U \times\left[\left(\int_{-\infty}^{-a} \xi_{n_{x}} \xi_{r_{x}} d x+\int_{a}^{\infty} \xi_{n_{x}} \xi_{n_{x}} d x\right)\left(\int_{-\infty}^{-a} \psi_{n_{y}} \psi_{n_{y}^{\prime}} d y+\int_{a}^{\infty} \psi_{n_{y}} \psi_{n_{y}^{\prime}} d y\right)\right]
\end{aligned}
$$

This matrix is independent of $k_{e z}$, which appears in Eq. (18). In this case, the exact Hamiltonian matrix is,

$$
\begin{aligned}
& \left\langle\hat{n}_{x}, \hat{n}_{y}, \tilde{n}_{z}|H| \hat{n}_{x}^{\prime}, \hat{n}_{y}^{\prime}, \tilde{n}_{z}^{\prime}\right\rangle= \\
& \quad\left\{\left\langle n_{x}, n_{y}\left|H_{00}\right| n_{x}^{\prime}, n_{y}^{\prime}\right\rangle+\left\langle n_{x}, n_{y}\left|V^{\prime}\right| n_{x}^{\prime}, n_{y}^{\prime}\right\rangle+\hbar^{2} k_{e z}^{2} / 2 m^{*}\right\} \delta_{\tilde{n}_{z}, \tilde{n}_{z}^{\prime}}
\end{aligned}
$$

where $\left\langle n_{x}, n_{y}\left|H_{00}\right| n_{x}^{\prime}, n_{y}^{\prime}\right\rangle$ is the diagonal matrix of elements equal to $E_{00}$. In this equation, $k_{e}$ is linked to $\tilde{n}_{z}$ by Eq. (7) where, for large values of $\tilde{n}_{z}, \theta$ may be neglected. Otherwise, Eq. (6) is to be used.

The eigenvalues of $\mathrm{H}_{00}+\mathrm{V}$ are represented in Fig. 2(b) in the sector labeled $1 \mathrm{Ez}$. In total there are $4 \times 4=16$ states; many of them degenerate. According to Eq. (20), they are the threshold of a set of continuous states rising upwards. This is clearly represented in the lower energy state by a light gray rectangle, but it should be noted that a separate continuum emanates from each state in this plot sector.

The development of the 16 2-dimensional (2D) eigenfunctions on the $16\left|n_{x}, n_{y}\right\rangle$ states is presented in the Supplementary materials. The 3D eigenfunctions are in this case the product of the 2D eigenfunctions times the 1D extended (in/out phase harmonic) wave corresponding to $k_{e z}$.

A comparison with the position of the thresholds analyzed above when the Hamiltonian is $H_{0}$ is presented in the Supplementary materials. They are actually located much further above: the perturbation potential brings them down substantially. However, they approach but do not invade the barrier material bandgap. This may be a general rule that could be mathematically proven. We have not attempted to do it. The $1 \mathrm{st}$ order approximation gives closer values (see Supplementary materials) but in most cases not very much.

For the 1Ex and 1Ey sets, an analogous treatment is developed. In this case, there are $4 \times 2=8\left|n_{y}, n_{z}\right\rangle$ states and the same for $\left|n_{x}, n_{z}\right\rangle$. They appear in Fig. 2(b) and are the thresholds of series of continuous spectrum states. The development of the two- dimensional eigenstates in $\left|n_{y}, n_{z}\right\rangle$ the $E_{00}$ and $E_{10}$ and approximations can also be found in the Supplementary materials.

\subsubsection{Two dimensional extended $2 E$ states}

This subsection actually deals with three sets, 2Exy, 2Eyz and 2Ezx, corresponding respectively to bound states only in $z, x$ and $y$ and extended in the remaining variables. Let us examine first the 2Exy states. The eigenvalues of $H_{0}$ are

$$
\begin{aligned}
E_{0} & =E_{z, n_{z}}+\left(U+\hbar^{2} k_{e x}^{2} / 2 m^{*}\right)+\left(U+\hbar^{2} k_{e y}^{2} / 2 m^{*}\right)-U \\
& =E_{000}+\hbar^{2} k_{e x}^{2} / 2 m^{*}+\hbar^{2} k_{e y}^{2} / 2 m^{*}
\end{aligned}
$$

The subtracted $U$ sets the energy origin at the barrier material $\mathrm{CB}$ bottom. The second and third zero in the subindex signify that $k_{e x}$ and $k_{e y}$ are zero.

The matrix elements have now one bound functions and two extended; that is, they are, according to Eqs. (12) and (13),

$$
\begin{aligned}
& \left\langle\tilde{n}_{x}, \tilde{n}_{y}, \hat{n}_{z}\left|V^{\prime}\right| \tilde{n}_{x}^{\prime}, \tilde{n}_{y}^{\prime}, \hat{n}_{z}^{\prime}\right\rangle=\left\langle\hat{n}_{z}\left|V^{\prime}\right| \hat{n}_{z}^{\prime}\right\rangle \delta_{\tilde{n}_{x}, \hat{n}_{x}^{\prime}} \delta_{\tilde{n}_{y}, \tilde{n}_{y}^{\prime}}, \quad \text { where } \\
& \left\langle\hat{n}_{z}\left|V^{\prime}\right| \hat{n}_{z}^{\prime}\right\rangle=-U\left[\int_{-a}^{a} \zeta_{n_{z}} \zeta_{n_{z}^{\prime}} d z\right]-2 U\left[\left(\int_{-\infty}^{-a} \zeta_{n_{z}} \zeta_{n_{z}^{\prime}} d x+\int_{n}^{\infty} \zeta_{n_{z}} \zeta_{n_{z}^{\prime}} d x\right)\right]
\end{aligned}
$$

This matrix is independent of the $k_{e} s$, which appear in Eq. (21).

With the exact model for 2Exy states, the Hamiltonian matrix development is

$$
\begin{aligned}
& \left\langle\tilde{n}_{x}, \tilde{n}_{y}, \hat{n}_{z}|H| \tilde{n}_{x}^{\prime}, \tilde{n}_{y}^{\prime}, \hat{n}_{z}^{\prime}\right\rangle \\
& \quad=\left\{\left\langle n_{z}\left|H_{000}\right| n_{z}^{\prime}\right\rangle+\left\langle n_{z}\left|V^{\prime}\right| n_{z}^{\prime}\right\rangle+\hbar^{2} k_{e x}^{2} / 2 m^{*}+\hbar^{2} k_{e y}^{2} / 2 m^{*}\right\} \delta\left(k_{e x}, k_{e x}^{\prime}\right) \delta\left(k_{e y}, k_{e y}^{\prime}\right)
\end{aligned}
$$

where $\left\langle n_{z}\left|H_{000}\right| n_{z}^{\prime}\right\rangle$ is the diagonal matrix of elements of $E_{000}$. The eigenvalues of $\mathrm{H}_{000}+V$ are represented in Fig. 2(b) in the sector labeled 1Exy. For $\left|n_{z}\right\rangle$, there are only 2 states, which are the threshold of a set of continuous states rising upwards (medium gray rectangles). The development of the two 1D eigenfunctions on the two $\left|n_{z}\right\rangle$ states is presented in the Supplementary materials. The $3 \mathrm{D}$ eigenfunctions are in this case the product of the $1 \mathrm{D}$ eigenfunctions times two $1 \mathrm{D}$ extended (in/ out phase harmonic) wave corresponding to the $k_{e x}$ and $k_{e y}$ vectors.

The same arguments may be applied to the 2Eyz and 2Ezx where the bound states are $\left|n_{x}\right\rangle$ and $\left|n_{y}\right\rangle$ respectively. In this case, there are four states and four levels, which are represented in the corresponding sector of the Fig. 2(b) plot. Again the lowering of the $H_{000}$ energy levels is very substantial, more than in the $1 \mathrm{E}$ sets; however, the levels do not penetrate into the bandgap, even though for the 2Eyz and 2Ezx sets they approach it closely. Details can be found in the Supplementary materials.

\subsection{4. $3 E$ set of three-dimensional extended states}

The eigenvalues of $H_{0}$ are,

$$
\begin{aligned}
E_{0} & =\left(U+\hbar^{2} k_{e x}^{2} / 2 m^{*}\right)+\left(U+\hbar^{2} k_{e x}^{2} / 2 m^{*}\right)+\left(U+\hbar^{2} k_{e z}^{2} / 2 m^{*}\right)-U \\
& =2 U+\hbar^{2} k_{e x}^{2} / 2 m^{*}+\hbar^{2} k_{e y}^{2} / 2 m^{*}+\hbar^{2} k_{e z}^{2} / 2 m^{*}
\end{aligned}
$$

The subtracted $U$ sets the energy origin at the barrier material $\mathrm{CB}$ bottom. In addition, taking into account Eq. (13), the perturbation matrix becomes very simple because

$$
\left\langle\tilde{n}_{x}, \tilde{n}_{y}, \hat{n}_{z}\left|V^{\prime}\right| \tilde{n}_{x}^{\prime}, \tilde{n}_{y}^{\prime}, \hat{n}_{z}^{\prime}\right\rangle=-2 U \delta_{\tilde{n}_{x}, \tilde{n}_{x}^{\prime}} \delta_{\tilde{n}_{y}, \tilde{n}_{y}^{\prime}} \delta_{\tilde{n}_{z}, \tilde{n}_{z}^{\prime}}
$$

In consequence,

$$
\begin{aligned}
\left\langle\tilde{n}_{x}, \tilde{n}_{y}, \hat{n}_{z}|H| \tilde{n}_{x}^{\prime}, \tilde{n}_{y}^{\prime}, \hat{n}_{z}^{\prime}\right\rangle= & \left(\hbar^{2} k_{e x}^{2} / 2 m^{*}+\hbar^{2} k_{e y}^{2} / 2 m^{*}\right. \\
& \left.+\hbar^{2} k_{e z}^{2} / 2 m^{*}\right) \delta_{\tilde{n}_{x}, \tilde{n}_{x}^{\prime}} \delta_{\tilde{n}_{y}, \tilde{n}_{y}^{\prime}} \delta_{\tilde{n}_{z}, \tilde{n}_{z}^{\prime}}
\end{aligned}
$$

which is the same as if the QD were absent although the wavevectors are not plane waves but the product of three 1D 
extended (in/out phase harmonic) wave corresponding to the $k_{e x}$, $k_{e y}$ and $k_{e z}$ vectors. Again, in this case, there is a substantial reduction of the $H_{0}$ eigenvalues: $2 U$ for all of them; however, they do not penetrate into the bandgap, but reach its upper edge. In Fig. 2 they are represented by the dark gray zone.

In the box-shaped QD, the continuous spectrum is heavily degenerated (as is visible in Fig. 2) and a richer wavefunction structure is possible. For instance, a virtual bound state (OE) may be combined with $1 \mathrm{E}, 2 \mathrm{E}$ and $3 \mathrm{E}$ states with the same energy, giving a rather complex eigenfunction that is (as any eigenfunction) a stationary state.

\section{Density of states}

For one-dimensional wavefunctions, the density of states per unit of length in the one-dimension $k$-space is $1 / \Delta k_{e} \cong 2 L / \pi$. Only positive values of $k$ are to be considered.

For a single QD, the density of $3 E$ states per unit of $\boldsymbol{k}$-space volume is $1 / \Delta k_{e x} \Delta k_{e y} \Delta k_{e z} \cong 8 L^{3} / \pi^{3}$. Let us assume that the density of QDs is $N_{Q D}$; the volume per QD is $1 / N_{Q D}$. It seems reasonable to select it in our calculations as the volume associated to one QD. We are assuming that the density is low enough as to prevent any influence of a QD on the others. Therefore, $1 / \Delta k_{e x} \Delta k_{e y} \Delta k_{e z} \cong$ $1 / N_{Q D} \pi^{3}$. Taking into account that there are $N_{Q D}$ states per unit of volume, the total density of states per unit $\boldsymbol{k}$ - and $\boldsymbol{r}$-space is $1 / \Delta k_{e x} \Delta k_{e y} \Delta k_{e z} \cong 1 / \pi^{3}$, independent of the density of QDs. The states from different QDs will have their $\boldsymbol{k}$-points intercalated in the continuum of $\boldsymbol{k s}$.

Reproducing the usual calculation, the number of states per unit of volume for energy below a given value is $M_{3 E}(E)=2 \times$ $(4 / 3) \pi\left(2 m^{*} E / h^{2}\right)^{3 / 2} / 8 \pi^{3}$ where the first factor of 2 is due to the spin degeneracy and the 8 in the denominator refers to volume being of a sphere octant. The expression for the density of states per unit of energy and volume is

$g_{3 E}(E)=\left(2 m^{*} / h^{2}\right)^{3 / 2} \sqrt{E} / 2 \pi^{2}$

This expression is also applicable for a bulk material without QDs: in this case the volume refers to the full sphere but the density is $(2 \pi)^{-3}$.

For a single $Q D$, the $2 \mathrm{E}$ density of states per $\boldsymbol{k}$-space unit of area is $1 / \Delta k_{e x} \Delta k_{e y} \cong 4 L^{2} / \pi^{2}$. Let us assume that the superficial density of states is $N_{Q D, x y}$. The surface per $Q D$ is $1 / N_{Q D, x y}$. It seems reasonable to select this as a surface associated to one $Q D$ (it is assumed it does not affect the others). Thus $1 / \Delta k_{e x} \Delta k_{e y} \cong$ $1 / N_{Q D, x y} \pi^{2}$. Taking into account that there are $N_{Q D}$ per unit of volume, the total density of states per unit of $\boldsymbol{k}$ - and $\boldsymbol{r}$-space volume is $1 / \Delta k_{e x} \Delta k_{e y} \cong N_{Q D} / N_{Q D, x y} \pi^{2}$ where $N_{Q D, x y} / N_{Q D}=l_{Q D z}$ is the separation of $\mathrm{QD}$ layers in the $z$ direction. This direction is the growth direction and $l_{O D z}$ is a process parameter. In other directions, it will be $l_{Q D x}=l_{Q D y}=\left(N_{Q D, x y}\right)^{-1 / 2}$.

The number of states per unit of volume for energies below a given value is $M(E)=2 \times \pi\left(2 m^{*} E / h^{2}\right) / 4 \pi^{2} l_{Q D}$ where the first factor of 2 is due to the spin degeneracy and the 4 in the denominator accounts for the fact that the surface under consideration is a circle quadrant. $E$ is to be counted from its threshold, which depends on the QN of the bound onedimensional state. The expression for the density of states per unit of energy and volume is

$g_{2 E}(E)=\left(m^{*} / \hbar^{2}\right) / \pi l_{Q D}$

which is independent of the energy. This is the well known behavior of quantum wells.

For a single $\mathrm{QD}$, the $1 \mathrm{E}$ density of states per unit $k$-space length is $1 / \Delta k_{e} \cong 2 L / \pi$. The length associated to a $Q D$ is $l_{Q D}$. Thus
Table 4

Integrated density of states for the $3 \mathrm{E}, 2 \mathrm{E}, 1 \mathrm{E}$ and $\mathrm{OE}$ sets in several energy intervals.

\begin{tabular}{lllll}
\hline States, $\mathrm{cm}^{-3} ; \mathrm{E}, \mathrm{eV}$ & $3 \mathrm{E}$ & $2 \mathrm{E}$ & $1 \mathrm{E}$ & $0 \mathrm{E}$ \\
\hline$M(0.5)$ & $8.09 \times 10^{18}$ & $7.68 \times 10^{17}$ & $1.09 \times 10^{17}$ & $9.77 \times 10^{15}$ \\
Sum states, $-0.5<E<0$ & 0 & 0 & 0 & $5.86 \times 10^{16}$ \\
Sum states, $0<E<0.5$ & $8.09 \times 10^{18}$ & $8.46 \times 10^{18}$ & $2.0 \times 10^{18}$ & $2.54 \times 10^{17}$ \\
\hline
\end{tabular}

$N_{Q D}=4.88281 \times 10^{15} \mathrm{~cm}^{-3} ; N_{Q D, x y}=3.90625 \times 10^{10} \mathrm{~cm}^{-2} ; l_{Q D, z}=8 \times 10^{-6} \mathrm{~cm}$.

$1 / \Delta k_{e} \cong l_{Q D} / \pi$. Taking into account that there are $N_{Q D}$ states per unit of volume, the total density of states per unit of $k$ - and $\boldsymbol{r}$ space volume is $1 / \Delta k_{e} \cong N_{Q D} l_{Q D} / \pi$. Note that $N_{Q D} l_{Q D z}=N_{Q D, x y}$, although $N_{Q D} l_{Q D x}=N_{Q D} l_{Q D y}=N_{Q D} / \sqrt{N_{Q D, x y}}$.

The number of states per unit of yolume for energies below a given value is $M(E)=2 \times\left(2 m^{*} E / h^{2}\right)^{1 / 2} N_{Q D} l_{Q D} / \pi$ where the first factor of 2 is due to the spin degeneracy. The expression for the density of states is

$g(E)=\left(2 m^{*} / h^{2}\right)^{1 / 2} N_{Q D} l_{Q D} / \pi \sqrt{E}$

which tends to infinity at the energy onset. This is the well known behavior of quantum wires.

Table 4 shows the integrated density of states per unit of volume for an interval energy interval of $0.5 \mathrm{eV}$. In the first row, the values of $M_{2 \mathrm{E}}$ and $M_{1 \mathrm{E}}$ are calculated for a single $\left|n_{z}\right\rangle$ or $\left|n_{x}, n_{y}\right\rangle$ state, respectively, for the energy interval ranging from the threshold to $0.5 \mathrm{eV}$ above it. $M_{\mathrm{OE}}$ is calculated for a single nondegenerated (besides the spin degeneracy that is taken into account) state. In the rows below all the states due to all the levels in the interval specified are calculated.

The density of states associated to the density-of-probability layers in the coordinate planes (2E states) is remarkably high as it is the density of states associated to the coordinate axes (1E states), exceeding or approaching the density of states of the bulk material ( $3 \mathrm{E}$ states). The totally bound states $(\mathrm{OE})$ are less abundant.

It is to be understood that the continuous states are strongly degenerated permitting a large variety of stationary wavefunctions formed by linear combination for a given energy of those described. In practice, almost all these states are empty of electrons.

\section{Spherical symmetry and the cubic case}

The current method of QD growth, consisting in growing InAs layers on a GaAs substrate in a molecular beam epitaxy (MBE) apparatus, tends to produce squat QDs. Some studies on QDs assume that they are spherical. This is a good approximation for colloidal QDs. However, the differences between squat, cubic and spherical QDs are substantial.

The mathematical treatment of spherical QDs can be found in textbooks (e.g. [20]: the case of a central potential). The eigenfunctions takes the form $\Phi(r, \theta, \varphi)=Y_{l}^{m}(\theta, \varphi) f_{l}(r)$ where the functions $Y_{l}^{m}(\theta, \varphi)$ are spherical harmonics, which are labeled by the angular and magnetic QNs $l$ and $m$ respectively, and the radial wavefunction $f_{l}(r)$ obeys to the radial equation. For a square potential well, the radial solution is the spherical Bessel function $j_{l}(k r)$. Bound states appear only for certain energies (those leading to fading solutions outside the well) below the potential wellhead. For energy above the wellhead, the spectrum is continuous and the asymptotic behavior of the solutions for large $r$ are spherical waves of the form $\sin (k r-\theta) / r$. No virtual bound states are found for a large variety of potential profiles, provided that they tend to zero (the wellhead energy) fast enough. 
Table 5

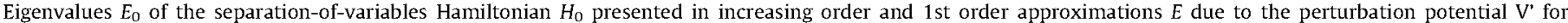
wavefunctions of the class $\mathrm{OE}$ (bound states). The energy origin is located at the barrier material $\mathrm{CB}$ bottom. The 1 st order approximation is exact.

\begin{tabular}{|c|c|c|c|c|c|c|c|c|}
\hline State & $|1,1,1\rangle$ & $|2,1,1\rangle$ & $|1,2,1\rangle$ & $|1,1,2\rangle$ & $|2,2,1\rangle$ & $|2,1,2\rangle$ & $|1,2,2\rangle$ & $|2,2,2\rangle$ \\
\hline$E_{0}(\mathrm{eV})$ & -0.0681 & 0.2105 & 0.2105 & 0.2105 & 0.4891 & 0.4891 & 0.4891 & 0.7677 \\
\hline$E(\mathrm{eV})$ & -0.0890 & 0.1385 & 0.1385 & 0.1385 & 0.2767 & 0.2767 & 0.2767 & 0.3728 \\
\hline
\end{tabular}

Smaller QDs are desirable for many applications and they have often been studied under the spherical symmetry. We analyze now a cubic box-shaped QD in which we set the current QD height $(2 \times 3 \mathrm{~nm})$ for the three parallelepiped edges and leave the remaining parameters unchanged. For the bound states we obtain Table 5. In this case, the 1st order approximation coincides with the exact solution. The exact eigenvectors are the separation-ofvariables solutions denoted in the table.

In contrast, the spherical QD with the same volume as the cube of $6 \mathrm{~nm}$ of side has a diameter of $7.4 \mathrm{~nm}$ and the $s$-state $(l=0)$ energy is $-0.0902 \mathrm{eV}$, rather close to the only below bandgap bound state energy of $-0.0890 \mathrm{eV}$ obtained for the cube. In the sphere this is the only bound state; neither a second s-state nor states with any other angular symmetry are present for the parameters used. Thus, the absence of virtual bound states is a singularity of the high symmetry of the spherical QD.

In the cube, there are also eigenfunctions that are extended in one dimension or in two dimensions and bound in the remaining ones. Their continuous spectrum starts well inside the barrier material CB. Values are not reported for lack of specific interest. These types of solutions are also missing in the spherical QDs.

Apparently, the spherical-wave solutions are missing in the cube. Actually this is not true. A spherical wave can be built as continuous linear combination of plane waves. All the $\boldsymbol{k}$-vectors of module $k$ are the components. In effect, let us analyze the function

$$
\begin{aligned}
\Phi(\boldsymbol{r}) & =\int \delta(|\boldsymbol{k}|-k) \exp (\boldsymbol{i k} \cdot \boldsymbol{r}) d^{3} k=2 \pi k^{2} \int_{0}^{\pi} \exp (i k r \cos \vartheta) \sin \vartheta d \vartheta \\
& =\frac{4 \pi k}{r} \sin (k r)
\end{aligned}
$$

where $\vartheta$ is the angle between the vectors $\boldsymbol{k}$ and $\boldsymbol{r}$ (spherical coordinates in the $\boldsymbol{k}$-space are used and the polar-axis is taken to be in the direction of $\boldsymbol{r}$ ). For the final result, the change of variables $\cos \vartheta=u$ has been made.

For large values of $r$ the function $\exp (i \boldsymbol{k} \cdot \boldsymbol{r})$ is also an eigenstate of the box-shaped potential well and the same happens with the function $\exp (-\boldsymbol{i} \boldsymbol{k} \cdot \boldsymbol{r})$. To prove it, let us go back to the one dimensional eigenfunctions,

$$
\begin{aligned}
e^{i k_{x} x} & =\frac{\left(e^{i \theta_{o}} \xi_{k, e}+i e^{i \theta_{e}} \xi_{k, o}\right)}{\cos \left(\theta_{0}-\theta_{e}\right)} \\
e^{-i k_{x} x} & =\frac{\left(e^{-i \theta_{0}} \xi_{k, e}-i e^{-i \theta_{e}} \xi_{k, o}\right)}{i \sin \left(\theta_{e}-\theta_{o}\right)}
\end{aligned}
$$

In the first octant (where all the space coordinates are positive) the exponential is developed in the eigenfunctions described above as ( $e$ and $o$ mean even and odd respectively)

$$
\begin{aligned}
e^{i k \cdot \boldsymbol{r}}= & \frac{e^{i\left(\theta_{x o}+\theta_{y o}+\theta_{z o}\right)}\left|k_{x, e}, k_{y, e}, k_{z, e}\right\rangle+i e^{i\left(\theta_{x o}+\theta_{y o}+\theta_{z e}\right)}\left|k_{x, e}, k_{y, e}, k_{z, o}\right\rangle}{\cos \left(\theta_{x o}-\theta_{x e}\right) \cos \left(\theta_{y o}-\theta_{y e}\right) \cos \left(\theta_{z o}-\theta_{z e}\right)} \\
& +\frac{i e^{i\left(\theta_{x o}+\theta_{y e}+\theta_{z o}\right)}\left|k_{x, e}, k_{y, o}, k_{z, e}\right\rangle+i e^{i\left(\theta_{x e}+\theta_{y o}+\theta_{z o}\right)}\left|k_{x, o}, k_{y, e}, k_{z, e}\right\rangle}{\cos \left(\theta_{x o}-\theta_{x e}\right) \cos \left(\theta_{y o}-\theta_{y e}\right) \cos \left(\theta_{z o}-\theta_{z e}\right)} \\
& -\frac{e^{i\left(\theta_{x o}+\theta_{y e}+\theta_{z e}\right)}\left|k_{x, e}, k_{y, o}, k_{z, o}\right\rangle+e^{i\left(\theta_{x e}+\theta_{y o}+\theta_{z e}\right)}\left|k_{x, o}, k_{y, e}, k_{z, o}\right\rangle}{\cos \left(\theta_{x o}-\theta_{x e}\right) \cos \left(\theta_{y o}-\theta_{y e}\right) \cos \left(\theta_{z o}-\theta_{z e}\right)} \\
& -\frac{e^{i\left(\theta_{x e}+\theta_{y e}+\theta_{z o}\right)}\left|k_{x, o}, k_{y, o}, k_{z, e}\right\rangle i e^{i\left(\theta_{x e}+\theta_{y e}+\theta_{z e}\right)}\left|k_{x, o}, k_{y, o}, k_{z, o}\right\rangle}{\cos \left(\theta_{x o}-\theta_{x e}\right) \cos \left(\theta_{y o}-\theta_{y e}\right) \cos \left(\theta_{z o}-\theta_{z e}\right)}
\end{aligned}
$$

In other octants the linear combination is somewhat different insofar as the de-phasing angles change the sign for the negative coordinate. For the wave plane traveling backwards, a slightly different linear combination is to be built.

Once the plane wave is built, a linear combination of them leads to the spherical wave of Eq. (30). Notice that, in the context of this paper, the $k$ s are restricted to the first octant in the $\boldsymbol{k}$-space. However, the same result is obtained if the linear combination $\exp (\boldsymbol{i k} \boldsymbol{r})+\exp (-\boldsymbol{i} \boldsymbol{k} \boldsymbol{r})$ is integrated.

As is well known, plane waves can also be obtained as a linear combination of spherical waves (see e.g., [20]). Thus the main difference between the spherical and box-shaped squarepotential-well QDs is that the latter presents virtual OE bound states and linear and planar $1 \mathrm{E}$ and $2 \mathrm{E}$ extended states, all absent in the spherical QD. It has to be stressed that the solutions we are presenting here are exact solutions for the model described. The same model is used for the spherical QD with only the change of shape.

\section{Conclusions}

In this paper, a simple Hamiltonian, associated to QDs characterized by a square (in the energy axis) potential well with the shape of a parallelepiped, is solved exactly. The effective mass is taken to be the same outside and inside the QD. The adequacy of this model to a real QD may have several shortcomings, as described in the introduction, but, nevertheless, it is thought to approximately describe the QD energy spectrum in the vicinity of the $\mathrm{CB}$.

The exact solution is obtained by developing the Hamiltonian in a basis of separation-of-variables solutions already used in preceding papers. Then the Hamiltonian matrix is diagonalized and the eigenvalues and eigenvectors are obtained.

Considering only the mentioned basis, several sets of states are found: with the wavefunctions bound in all the three dimensions (OE states), bound in two dimensions and extended in one (1E states), bound in one dimension and extended in two (2E states) and extended in all three dimensions (3E states). The latter resemble the plane waves of the barrier material without any QD and produce the same density of states. The wavefunction states extended in one or two dimensions form filaments and sheets of high electron density of probability (if filled with electron), directed along the three coordinate axes and planes. In this respect, they respectively present a density of states similar to those of the quantum wires and wells, although in the $\mathrm{QD}$ case they are inside the barrier material $C B$ while the quantum wire and quantum wells start inside the bandgap. We think that this fact has not been clearly reported so far, perhaps due to the clean results found in QDs with spherical symmetry. However, it has to be stressed that the presence of these states derives from the solution of a mathematical problem that is the same in all but shape. As matter of fact, the density of states associated to the wire and well type states is high and comparable to that of the all-extended wavefunction states.

These virtual bound states are of big importance because they are an effective means of photon absorption and emission between the ordinary bound states within the bandgap and the 
conduction band [32]. Transitions to the ordinary 3E states and to the $2 \mathrm{E}$ states are forbidden $[25,28]$. Transitions to the $1 \mathrm{E}$ filamentary states are, however, permitted and may be the second path for the photonic interaction.

The $3 \mathrm{E}$ extended states resemble the free electron traveling waves, but spherical wavefunctions can be obtained from these plane waves (and conversely). In fact, the heavily degenerate continuous spectrum of the box-shaped QD allows for a large variety of stationary states; e.g., plane waves, spherical waves or a combination of those with bound $(\mathrm{OE})$, filamentary (1E) and planar (2D) states.

The use of spherical waves for the evaluation of the optical elements of matrix, which is the natural choice in spherical symmetry, is much more complicated than the use of the separation of variables wavefunctions, which is the natural choice for box-shaped QDs.

The exact solution is very similar in energy and wavefunction to those obtained in the separation-of-variables approximation for low energy bound states, in particular for those lying within the bandgap. The differences are strong, in particular in energy, for states high in the barrier material $C B$. This is interesting because it validates the several studies on sub-bandgap photon absorption among bound states based on the separation-ofvariables approximation. On the contrary, the few studies on bound to one-dimensional $(0 \mathrm{E} \rightarrow 1 \mathrm{E})$ extended states (the only bound to extended transitions with non-zero elements of matrix) must be revisited. In all cases, the use of the first order perturbation, which is easy to handle, may be of practical value even if not totally exact. However, the highest energies in the separation-ofvariables approximation are severely decreased in the exact calculation (or even in the first order approximation).

\section{Acknowledgment}

This work has been partially supported by the EU Grant NGCPV (283798) and the Madrid Regional Government Grant NUMANCIA-2 (S2009/ENE1477). EA acknowledges a Juan de la Cierva Post-Doctoral Fellowship (JCI-2011-10639) from the Spanish Ministry of Science.

\section{Appendix A. Supplementary materials}

Supplementary materials associated with this article can be found in the online version at http://dx.doi.org/10.1016/j.physb.2012.12.047.

\section{References}

1] L.W. Wang, A. Zunger, J. Phys. Chem. 98 (1994) 2158.

[2] L.C.L.Y. Voon, M. Willatzen, The k.p Method, Springer, Berlin, 2009.

[3] O. Stier, M. Grundmann, D. Bimberg, Phys. Rev. B 59 (1999) 5688.

[4] C. Pryor, Phys. Rev. B 57 (1998) 7190.

[5] V. Popescu, G. Bester, A. Zunger, Appl. Phys. Lett. 95 (2009) 023108.

[6] V. Popescu, G. Bester, M.C. Hanna, A.G. Norman, A. Zunger, Phys. Rev. B 78 (2008) 205321

7] S. Tomic, A. Marti, E. Antolin, A. Luque, Appl. Phys. Lett. 99 (2011) 053504

[8] A. Luque, A. Martí, Phys. Rev. Lett. 78 (1997) 5014

[9] A. Luque, A. Martí, Prog. Photovoltaics Res. Appl. 9 (2001) 73.

[10] A. Luque, A. Marti, Adv. Mater. 22 (2009) 160

[11] A. Luque, A. Marti, C. Stanley, Nat. Photonics 6 (2012) 142

[12] H.C. Liu, J.Y. Duboz, R. Dudek, Z.R. Wasilewski, S. Fafard, P. Finnie, Physica E 17 (2003) 631

[13] K.W. Berryman, S.A. Lyon, M. Segev, Appl. Phys. Lett. 70 (1997) 1861.

[14] G. Breit, E. Wigner, Phys. Rev. 49 (1936) 0519.

[15] G. Bastard, U.O. Ziemelis, C. Delalande, M. Voos, A.C. Gossard, W. Wiegmann, Solid State Commun. 49 (1984) 671

[16] R. Ferreira, G. Bastard, Nanoscale Res. Lett. 1 (2006) 120.

[17] X.G. Peng, L. Manna, W.D. Yang, J. Wickham, E. Scher, A. Kadavanich, A.P. Alivisatos, Nature 404 (2000) 59.

[18] A.I. Ekimov, F. Hache, M.C. Schanneklein, D. Ricard, C. Flytzanis, I.A. Kudryavtsev, T.V. Yazeva, A.V. Rodina, A.L. Efros, J. Opt. Soc. Am. B Opt. Phys. 10 (1993) 100.

[19] E. Antolín, A. Martî, A. Luque, Proceedings of the 37th Photovoltaic Specialists Conference, IEEE, Seattle, 2011

[20] A. Messiah, Mêcanique Quantique, Dunod, Paris, 1960

[21] A. Martí, L. Cuadra, A. Luque, Proceedings of the 28th IEEE Photovoltaics Specialists Conference, IEEE, New York, 2000, p. 940.

[22] P.G. Linares, A. Marti, E. Antolin, A. Luque, J. Appl. Phys. 109 (2011) 014313.

[23] Q. Shao, A.A. Balandin, A.I. Fedoseyev, M. Turowski, Appl. Phys. Lett. 91 (2007) 163503.

[24] O.L. Lazarenkova, A.A. Balandin, J. Appl. Phys. 89 (2001) 5509.

[25] A. Luque, A. Marti, E. Antolin, P. Garcia-Linares, Sol. Energ Mater. Sol. Cell. 94 (2010) 2032.

[26] A. Luque, A. Marti, E. Antolín, P.G. Linares, I. Tobias, I. Ramiro, AIP Adv. 1 (2011) 022125

[27] A. Luque, A. Marti, E. Antolín, P.G. Linares, I. Tobías, I. Ramiro, E. Hernandez, Sol. Energ Mater. Solar Cell. 95 (2011) 2095

[28] A. Luque, A. Marti, A. Mellor, D.F. Marron, I. Tobias, E. Antolín, Prog. Photovoltaics (2012), http://dx.doi.org/10.1002/pip.1250.

[29] A. Luque, A. Mellor, E. Antolin, P.G. Linares, I. Ramiro, I. Tobias, A. Marti, Sol. Energ. Mater. Sol. Cell. 103 (2012) 171

[30] E. Antolín, A. Marti, C.D. Farmer, P.G. Linares, E. Hernández, A.M. Sánchez, T. Ben, S.I. Molina, C.R. Stanley, A. Luque, J. Appl. Phys. 108 (2010) 064513.

[31] S. Datta, Quantum Phenomena, Addison Wesley, Reading, Massachusetts, 1989. ISBN 0-201-07956-9.

[32] A. Mellor, A. Luque, I. Tobias, A. Marti, Appl. Phys. Lett. 101 (2012) 133909. 